\title{
Use of the RoboFlag synthetic task environment to investigate workload and stress responses in $\mathrm{UAV}$ operation
}

\author{
Svyatoslav Guznov • Gerald Matthews • \\ Gregory Funke • Allen Dukes
}

Published online: 13 April 2011

(C) Psychonomic Society, Inc. 2011

\begin{abstract}
Use of unmanned aerial vehicles (UAVs) is an increasingly important element of military missions. However, controlling UAVs may impose high stress and workload on the operator. This study evaluated the use of the RoboFlag simulated environment as a means for profiling multiple dimensions of stress and workload response to a task requiring control of multiple vehicles (robots). It tested the effects of two workload manipulations, environmental uncertainty (i.e., UAV's visual view area) and maneuverability, in 64 participants. The findings confirmed that the task produced substantial workload and elevated distress. Dissociations between the stress and performance effects of the manipulations confirmed the utility of a multivariate approach to assessment. Contrary to expectations, distress and some aspects of workload were highest in the low-uncertainty condition, suggesting that overload of information may be an issue for UAV interface designers. The strengths and limitations of RoboFlag as a methodology for investigating stress and workload responses are discussed.
\end{abstract}

Keywords Workload·Stress · Computer simulators

Technological advancements are creating new challenges in human factors. Computer systems are increasingly trans-

\footnotetext{
S. Guznov $(\bowtie) \cdot$ G. Matthews

Department of Psychology, University of Cincinnati,

5140 H Edwards Hall 1,

Cincinnati, OH 45221, USA

e-mail: guznovs@mail.uc.edu

G. Funke $\cdot$ A. Dukes

Air Force Research Laboratory, Wright Patterson Air Force Base,

Dayton, $\mathrm{OH}$, USA
}

forming work demands in fields including industry, transportation, and the military. Changing work demands, in turn, may influence operator workload and stress, sometimes unexpectedly. For example, studies of driver behavior have shown that some forms of vehicle automation may lower workload but increase stress (Saxby, Matthews, Hitchcock, \& Warm, 2007) and impair situation awareness and speed of response to traffic events (Young \& Stanton, 2007). Findings of these kinds demonstrate the need for methods to evaluate stress and workload response for novel, complex operational tasks and activities. As the driver behavior research shows, it is important that these methods be capable of discriminating the impact of the task on stress, workload, and performance. In this article, we focus on unmanned aerial vehicles (UAVs) as an exemplar of a new technology whose effects on stress and workload are not well understood.

UAVs are extensively used for both military and civilian purposes (Mouloua, Gilson, \& Hancock, 2003; Parasuraman, Galster, \& Miller, 2003). For example, UAVs are frequently employed in surveillance and intelligence tasks. Although UAVs are best known from the military setting, it is likely that their civilian applications will multiply (McCarley \& Wickens, 2005). For example, UAVs may be used in fire-fighting, monitoring crops and wildlife, and search-and-rescue missions. The use of UAVs protects the human operator, but their control is a challenging supervisory task. UAV operators must control the trajectory of the craft, choose appropriate UAV automated functions, operate weapons, and evaluate risks. These cognitive demands may result in increased workload, stress, and reduced situational awareness (Parasuraman et al., 2003; Sterling \& Perala, 2007). Understanding the influences of workload and stress factors is becoming increasingly important due to the variety of environmental settings in which UAVs are used, both in the military and civilian 
sectors. The present study aimed to explore a new methodology for evaluating stress and workload responses using a laboratory task resembling UAV operation.

McCarley and Wickens (2005) identified several major human factors issues in UAV control, including automation, interface design, team organization, and team training. These are issues commonly encountered in a variety of other human factors domains as well. By contrast, there has been rather limited attention paid to stress and workload. There is a consensus that operators may need to deal with high workload, stress, and fatigue (Hancock, Mouloua, Gilson, Szalma, \& Oron-Gilad, 2007; Mouloua et al., 2003; Tvaryanas \& MacPherson, 2009). The multitasking nature of UAV operation in particular potentially poses high workload demands (Mouloua, Gilson, Krig, \& Hancock, 2001). However, two methodological issues must be confronted in order to make further progress. First, Mouloua et al. (2003) list numerous different sources of task load, which may be loosely divided into "input" (e.g., maintaining situation awareness) and "output" (e.g., manual control of vehicle) factors. Second, operator response to task load is likely to be multidimensional. Although stress is typically seen as an outcome of high workload, relationships between these constructs are complex. High workloads may not be highly stressful if the operator remains interested and engaged in the task, and low workload may be stressful if the task is monotonous (Hancock \& Szalma, 2003; Matthews, Warm, Reinerman, Langheim, \& Saxby, 2010). Furthermore, "stress" is itself a multidimensional construct that can be divided into different components, including emotional distress, worry, and loss of task engagement (Matthews et al., 2002). Workload may be differentially related to different components of the stress response, so new methods are needed for in-depth assessments of operator response to differing configurations of UAV tasks.

A synthetic task environment could be a valuable tool for investigating stress and workload in UAV operation. Synthetic task environments are defined by Martin, Lyon, and Schreiber (1998, p. 123) as "research tasks constructed by systematic abstraction from a corresponding real-world task." Cooke and Shope (2004) specified that while a synthetic task environment is often not identical to the target environment, it should capture the main characteristics of the task. A valid task environment should capture the cognitive components of the task and have sufficient face validity. Finally, the task should have a flexible architecture in order to facilitate various condition manipulations. Specifically, a computer-based task that imposed significant workload and stress might be an efficient compromise between a valid representation of the realworld environment and the experimental control afforded by laboratory tasks.
The RoboFlag simulated environment (D'Andrea \& Babish, 2003) has been used as a test bed for various behavioral studies involving the control of multiple robots by a single operator or a team of operators (Campbell et al., 2005; Parasuraman et al., 2003). The simulation is similar to the game Capture the Flag. Two opposing players or teams of players (Red Team and Blue Team) control multiple robots. The robots of the Red Team are controlled by the computer and represented by red squares $(3 \mathrm{~b}$ in Fig. 1), while the robots of the Blue Team are represented by blue circles ( $3 \mathrm{a}$ in Fig. 1). Each player's robots have a yellow sector around them that graphically represents the robot's area of visual view, similar to radar (4 in Fig. 1). Enemy robots become visible only when they move into that view. Otherwise, players are not able to see the opponent's robots. The team goal is to capture as many of the opponents' flags ( $5 \mathrm{a}$ or $5 \mathrm{~b}$ ) as possible and bring them back to their home territory (1a or $1 \mathrm{~b}$ ), while protecting their own flag.

The RoboFlag game appears to meet Cooke and Shope's (2004) criteria. Piloting a UAV is a form of robot operation (Hancock et al., 2007). The game captures its primary requirement of controlling multiple, partially automated robots. Tasks involving UAVs often require capturing or destroying a specified target while avoiding being destroyed by an enemy. RoboFlag also meets Cooke and Shope's last criterion of being a flexible experimenter tool because it facilitates different game scenarios. For example, it allows for changes in robot parameters (e.g., speed, sensor angle and radius, or fuel limit) and in the number of robots and their field coordinates.

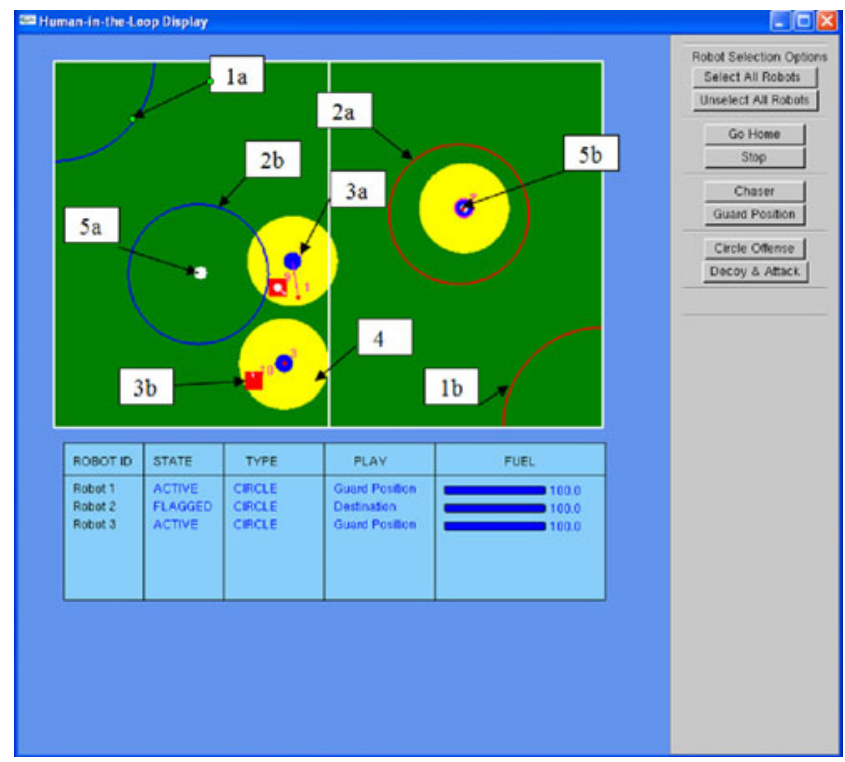

Fig. 1 RoboFlag game player screen 
Several studies have used RoboFlag as a means for investigating UAV team performance, interface design, and the design of the algorithm for the robots' behavior. Parasuraman et al. (2003) tested the effects of environmental uncertainty and game strategies on performance. Squire, Trafton, and Parasuraman (2006) investigated the problem of switching between tasks in multiple UAV environments. Finally, Funke, Galster, Nelson, and Dukes (2006) explored the possibility of using Internet instant messaging as an alternative to oral communication while controlling multiple UAVs.

RoboFlag is also potentially useful in investigating how UAV system properties affect the operator's stress response. Much research on operator stress has focused on environmental stress factors, but the cognitive demands of the task may also influence stress. Matthews et al. (2002; Matthews et al., 2006) proposed that taskinduced stress must be evaluated across multiple dimensions of affective, motivational, and cognitive states. Their dimensional model identifies the three higher-order stress state factors of task engagement, distress, and worry. Different sources of cognitive load elicit different patterns of state change across these factors. For example, vigilance tasks produce a substantial decrease in task engagement, whereas high working memory demands elevate distress, among other state changes (Matthews et al., 2006). However, the majority of this research has been directed toward simple laboratory tasks of limited relevance to UAV operation.

Thus far, studies of RoboFlag have investigated subjective workload, but not the operator's stress. Parasuraman, Galster, Squire, Furukawa, and Miller (2005) explored the impacts of environmental uncertainty and enemy engagement styles, using the NASA Task Load Index (Hart \& Staveland, 1988) to assess perceived workload. They found that increased environmental uncertainty decreased situational awareness and increased overall workload. Modes of team communication may also influence workload (Funke et al., 2006). According to Veverka and Campbell (2003), the speed of the robot in the RoboFlag simulation does not significantly affect operator workload. However, the manipulated speed parameter may not have been sufficiently different across experiments to reveal higher workload demands. Also, workload may be elevated if the robot is under- or overresponsive to the operator's control.

Distress might simply reflect overall workload. Studies of attention and working memory have shown that, overall, workload exerts a substantial effect on task-induced distress (Matthews et al., 2002), and so workload may also drive distress in the RoboFlag operator. However, it remains unclear whether stress response simply reflects total workload, or whether qualitative features of the task relevant to UAV operation, including uncertainty and controllability of the robot, exert more specific effects on the operator's mental state.

Two specific sources of task demand are uncertainty about key elements of the operating environment and the maneuverability or controllability of the vehicle's trajectory. In performance settings, uncertainty is often a major influence on stress and anxiety (Boyes \& French, 2010). Features of UAV operation seem apt to produce uncertainty over progress toward mission goals, such as detecting targets on the ground. UAVs have a limited visual range (Cummings \& Guerlain, 2007), so operators may be uncertain whether or not they have detected all relevant objects in the field of operation. UAV control monitors also have a limited image size and resolution, which may make target detection more difficult (van Erp, 2000). Indeed, imaging constraints such as the low frequency of temporal updates of the UAV camera (i.e., low refresh rate) may elevate operators' workload as well (van Erp \& van Breda, 1999). The nature of the terrain (Schipani, 2003) and night conditions (Cummings \& Guerlain, 2007) are further factors that may elevate both uncertainty and workload.

Maneuverability refers to the ease with which the UAV may be guided using the manual controls provided by the user interface. Difficulties in controlling the craft as intended may elevate both workload and stress, given that frustration in using computer interfaces has been linked to a stress response (Ceaparu, Lazar, Bessiere, Robinson, \& Shneiderman, 2004). Disruption of motor vehicle control has proven to be an effective stressor in research on simulated driving. Funke, Matthews, Warm, and Emo (2007) introduced wind gusts and reduced surface friction (ice) into the drive, so that the vehicle was episodically unresponsive to the driver's use of the steering wheel. Diminished vehicle control led to increased distress and workload. In the UAV context, Mouloua et al. (2003) pointed out that manual control may be disrupted by time delays in the transmission of information via satellite and by breaks in transmission. Response delays as short as $1 \mathrm{~s}$ may disrupt control of the aircraft (Worsch et al., 1996). It is plausible that these disruptions could be a source of stress for the operator.

This study aimed to evaluate the utility of RoboFlag as a tool for investigating stress and workload responses to manipulations of uncertainty and maneuverability. These manipulations were chosen to represent two potential types of influence on stress response in the UAV operator: constraints on information sampling, and disruption of the control characteristics of the vehicle. As previously discussed, one of the methodological challenges for workload assessment methods is that stress and workload responses are multidimensional and may be dissociated from each other, as well as from performance (Matthews et 
al., 2002; Wickens \& Hollands, 2000). Thus, the primary aim of the study was to investigate whether the RoboFlag simulated environment was capable of eliciting dissociations between performance, workload, and subjective stress responses, using two different workload manipulations. If RoboFlag can be used to delineate specific patterns of performance-workload-stress response for a given manipulation, then it may be useful for evaluating the operational significance of task load manipulations.

Maneuverability was defined here as a compound factor, related to both the robot's speed and its acceleration. Uncertainty was manipulated as a compound factor that depended on the robot's sensor view angle and sensor radius. It was expected that both high maneuverability (faster, more controllable robots) and lower uncertainty (more information on enemy robots) would improve performance. Both low maneuverability (disruption of operator control) and increased uncertainty over the locations of enemy robots were broadly expected to elevate workload and distress. Previous work on UAV operation has rarely attempted to discriminate multiple components of the workload/stress response. On an exploratory basis, we aimed to test whether the two manipulations elicited similar or different patterns of response across overall performance, six components of workload (Hart \& Staveland, 1988), and three fundamental stress state factors (Matthews et al., 2002).

\section{Method}

\section{Participants}

A total of 64 undergraduate students from the University of Cincinnati (32 men, 32 women) participated in the study to fulfill a credit requirement. Participants ranged in age from 18 to 26 years $(M=21.34, S D=1.6)$. They had normal or corrected-to-normal vision.

\section{Design}

The experiment employed a $2 \times 2$ (Uncertainty $\times$ Maneuverability) mixed-model design. Uncertainty was a within-subjects factor with two levels: high uncertainty (HU) and low uncertainty (LU). The Uncertainty factor was manipulated by varying the simulated robots' sensor view angles and sensor radii to influence the field of view (Fig. 2). Maneuverability was a between-subjects factor with two levels: high maneuverability (HM) and low maneuverability (LM). It was manipulated by varying the robots' speed and acceleration to influence their ease of control. This factor was manipulated between subjects because of concerns about difficulties in the transfer of control skills across the two maneuverability conditions. The dependent variables for this study were performance in the RoboFlag computer game, subjective stress, and subjective workload. The order of the uncertainty and maneuverability conditions was counterbalanced to minimize practice and transfer effects. Therefore, four groups of 16 participants performed tasks ordered as represented in Table 1.

\section{Materials and apparatus}

A PC running the RoboFlag program featured a $2.2-\mathrm{GHz}$ Pentium 4 dual-core processor, a 19-in. monitor with a screen resolution of $1,440 \times 900$ pixels, and a dual-head $512-\mathrm{Mb}$ video card. The player's monitor showed the RoboFlag game screen in the HU and LU conditions (Fig. 2).
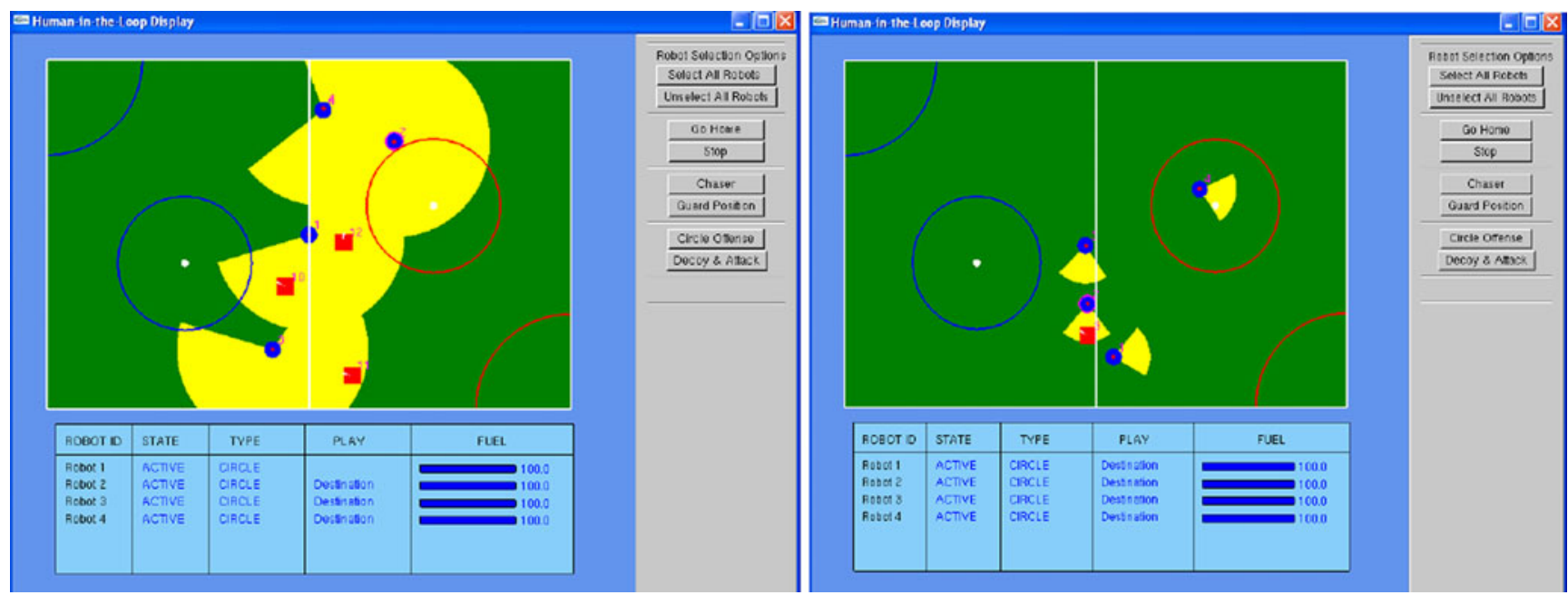

Fig. 2 RoboFlag game screenshots for the low-uncertainty (left) and high-uncertainty (right) conditions 
Table 1 Experimental conditions

\begin{tabular}{lll}
\hline Group & Task Phase 1 & Task Phase 2 \\
\hline 1 & HM-HU & HM-LU \\
2 & HM-LU & HM-HU \\
3 & LM-HU & LM-LU \\
4 & LM-LU & LM-HU \\
\hline
\end{tabular}

The RoboFlag 2.1 computer software (D'Andrea \& Babish, 2003) was used to simulate the multiple-UAV control environment, with each robot representing a single UAV. The participants controlled four robots of the Blue Team playing against the computer, which controlled six robots of the Red Team. The participants' goal was to capture the Red Team's flag and bring it back to their home base, while protecting their own flag by "tagging" enemy robots. To control a robot, the player first selected it via a mouse left-click and then moved the mouse pointer to the desired robot destination point by right-clicking the location. Robots were programmed to move to a selected destination point by the shortest straight line possible while avoiding obstacles. Each player's robots had yellow sectors around them that graphically represented the robots' visual view areas, similar to radar. Outside of the view area, players were not able to detect enemy robots. Once the enemy robot entered that view area, it became visible as a red square and could be intentionally tagged. To tag an enemy robot, players needed to collide one of their own robots with it in the player's half of the game field. If the collision happened in the enemy's half of the field, the player's robot would become tagged. A tagged robot returned to its home base. After its return, it was "repaired" and ready to be controlled again.

To capture the enemy flag, the player had to bring a robot into contact with the white circle, which indicated the enemy's flag (5a or 5 b of Fig. 1), and then return the robot to the player's home base without being tagged by the enemy. The player scored one point every time the flag was captured and one more if it was brought safely to the home territory. In addition, players scored every time they tagged the computer's robots. The RoboFlag software recorded primary task performance data including the number of flags captured, the number of flags brought home, and the number of opponents tagged.

Dundee Stress State Questionnaire (DSSQ) The Matthews et al. (2002) psychometric model of states identifies 11 correlated first-order dimensions, which support three broader second-order factors. The short version of the DSSQ (Matthews, Emo, \& Funke, 2005) assesses these three factors - that is, subjective engagement, distress, and worry states experienced pre- and posttask. Engagement refers to the qualities of energy, motivation, and concentration. Distress is defined by feelings of tension, positive hedonic tone, and confidence and control. Worry relates to self-focused attention, low self-esteem, and cognitive interference generated by the task and by personal concerns. The short DSSQ has been shown to be a valid alternative to the full version of the scale (Matthews et al., 2005).

Perceived Mental Workload (NASA-TLX) The NASA Task Load Index (NASA-TLX; Hart \& Staveland, 1988) requires the participant to rate task demands and his or her reactions to the task on six 100-point subscales that include Mental Demands, Physical Demands, Temporal Demands, Performance, Effort, and Frustration. A global workload score may be calculated by averaging the subscale ratings, but the focus of this study was on the individual subscale rating. The TLX is a standard and widely used measure of subjective workload (Wickens \& Hollands, 2000). Selfreported measures of workload were chosen for simplicity of administration and analysis.

\section{Procedure}

Prior to the beginning of the experimental task, participants completed informed consent forms and were randomly assigned to either the HM or the LM condition. Each participant filled out the pretask DSSQ, which allowed for assessing mood and feelings prior to the task. The items were simple to answer, and previous studies in which participants completed the DSSQ on multiple occasions have not shown any loss of reliability or validity across repeated testing (e.g., Matthews \& Campbell, 2010). Next, participants received a $10 \mathrm{~min}$ practice session. The participants were instructed about the RoboFlag game rules and their role in the task. In addition, they were shown how to control the robots, how to capture and bring home the flags, and how to tag enemy robots. After that, they were asked to practice these skills for $5 \mathrm{~min}$.

Upon completion of the practice phase, participants completed two task conditions; each condition was $6 \mathrm{~min}$ in duration. Pilot data suggested that this duration was sufficient for players to acquire a stable strategy for performance. A longer duration would have increased the likelihood of within-session changes in stress and fatigue.

After each game condition, participants were asked to rate their subjective workload and stress using computerbased versions of the DSSQ and NASA-TLX. After the experimental trials, participants were asked about their video game experience, expressed in number of hours of video games played per week; the mean of hours played 
was $4.1 \mathrm{~h}(S D=4.8)$. The two groups (HM and LM) did not differ significantly on this measure, $t(62)=1.67$, $p>.05$. The duration of the entire experiment was approximately $45 \mathrm{~min}$.

\section{Results}

The strategy for data analysis was as follows. First, we tested whether the two task parameters (uncertainty and maneuverability) influenced performance as expected, as a manipulation check. Next, we evaluated the effects of task parameters on the three dimensions of stress state, using an omnibus analysis, followed by planned comparisons. A similar analysis of workload data was also performed.

\section{Performance data}

RoboFlag overall players' performance A number of different performance indices are available from RoboFlag. These indices are affected both by the player's overall competence at the task and by strategy choices such as favoring offense over defense. The aim of the present study was to use uncertainty and maneuverability as means for influencing task demands, so investigation of strategic aspects of performance was beyond its scope. For this reason, we used a single performance metric that combined indices corresponding to the three key goals for the player: to capture more flags than the opponent, to bring home more flags than the opponent, and to tag enemy robots more frequently than the opponent. Overall performance was thus defined as the difference between the player's and computer's performance in number of flags captured, number of flags brought home, and enemy robots tagged.

The following formula was employed:

$$
\begin{aligned}
\text { Performance }= & \left(\text { Capture }_{\mathrm{P}}-\text { Capture }_{\mathrm{C}}\right) \\
& +\left(\text { Home }_{\mathrm{P}}-\text { Home }_{\mathrm{C}}\right)+\left(\text { Tags }_{\mathrm{P}}-\text { Tags }_{\mathrm{C}}\right),
\end{aligned}
$$

where Capture $_{P}$ is the number of flags captured by the participant during a trial, Capture ${ }_{C}$ is the number of flags captured by the computer, Home $e_{P}$ is the number of flags successfully brought to home territory by the participant, Home $_{C}$ is the number of flags successfully brought to home territory by the computer, Tags ${ }_{P}$ is the number of enemy robot tags initiated by the participant, and $\mathrm{Tags}_{\mathrm{C}}$ is the number of participant robot tags initiated by the computer. This formula provides a single metric that summarizes offensive (i.e., the differences in the numbers of flags captured and brought home) and defensive (i.e., the difference in the number of tags) performances. A 2 (uncertainty) $\times 2$ (maneuverability) mixed-model ANOVA revealed statistically significant main effects of uncertainty, $F(1,62)=17.21, p<.01, \eta_{\mathrm{p}}{ }^{2}=.22$, and maneuverability, $F(1,62)=16.97, p<.01, \eta_{\mathrm{p}}{ }^{2}=.21$. The results also revealed a significant interaction between them, $F(1,62)=$ 5.03, $p<.05, \eta_{\mathrm{p}}{ }^{2}=.07$. Participants in the HM condition $(M=15.22, S D=5.73)$ performed significantly better than participants in the LM condition $(M=12.74, S D=5.1)$, $t(62)=4.17, \quad p<.01$, but the effect of maneuverability was stronger under high uncertainty. Overall performance results are displayed in Figure 3.

\section{Subjective stress (DSSQ)}

Two kinds of question might be asked in evaluating the stress response to a complex real-world task such as UAV operation. First, we might ask whether the experience of the task is generally stressful, acknowledging that different missions will vary in stress response (Sterling \& Perala, 2007). Second, we may seek to unpack specific elements of the task and assess their contributions to stress response. To address these two issues, we conducted an omnibus analysis of the experimentally manipulated factors, followed by planned comparisons to differentiate effects of the task as a whole from those of the uncertainty manipulation.

Independent-samples $t$ tests were conducted for each of the pretest DSSQ factors to ensure the similarity of their perceived stress ratings before the task. No significant difference was revealed between the HM and LM groups.

The omnibus analysis was a $3 \times 2$ (Task Phase $\times$ Maneuverability) mixed-model ANOVA. Task Phase was a within-subjects factor consisting of the levels pretask, posttask low uncertainty, and posttask high uncertainty. Cell means and standard deviations are given in Table 2.

Separate ANOVAs were calculated for each DSSQ factor. The main effect of task phase was significant for task engagement, $F(2,124)=8.91, p<.01, \eta_{\mathrm{p}}{ }^{2}=.13$,

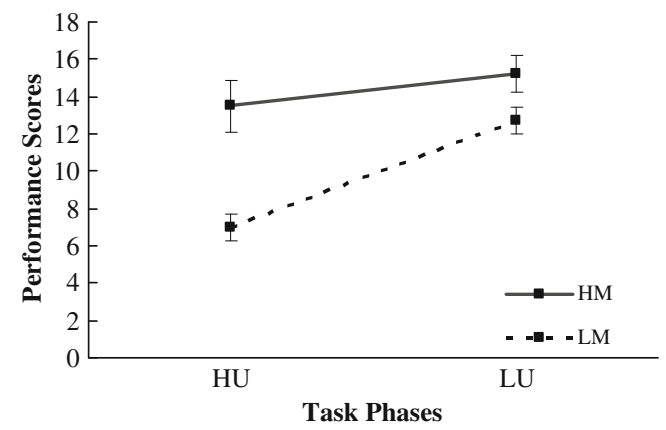

Fig. 3 Mean performance scores across task phases. Error bars are standard errors. HM, high maneuverability; LM, low maneuverability; HU, high uncertainty; LU, low uncertainty 
Table 2 Descriptive statistics for the DSSQ factors

\begin{tabular}{|c|c|c|c|c|c|}
\hline & & \multicolumn{2}{|l|}{ HM } & \multicolumn{2}{|l|}{ LM } \\
\hline & & $M$ & $S D$ & $M$ & $S D$ \\
\hline \multirow[t]{3}{*}{ Engagement } & Pretest & 21.47 & 4.19 & 20.50 & 3.66 \\
\hline & $\mathrm{HU}$ & 22.69 & 7.29 & 22.63 & 6.15 \\
\hline & $\mathrm{LU}$ & 24.00 & 4.35 & 24.00 & 5.58 \\
\hline \multirow[t]{3}{*}{ Distress } & Pretest & 9.06 & 3.74 & 9.84 & 3.82 \\
\hline & $\mathrm{HU}$ & 10.28 & 5.43 & 11.41 & 5.60 \\
\hline & LU & 11.69 & 6.30 & 13.09 & 4.42 \\
\hline \multirow[t]{3}{*}{ Worry } & Pretest & 10.19 & 5.09 & 11.56 & 7.09 \\
\hline & $\mathrm{HU}$ & 6.56 & 4.74 & 8.09 & 6.00 \\
\hline & LU & 6.50 & 5.41 & 7.19 & 5.30 \\
\hline
\end{tabular}

HM, high maneuverability; LM, low maneuverability; HU, high uncertainty; LU, low uncertainty.

distress, $F(2,124)=7.79, p<.05, \eta_{\mathrm{p}}^{2}=.11$, and worry, $F(2,124)=19.31, p<.01, \eta_{\mathrm{p}}^{2}=.3$. There were no significant main or interactive effects of maneuverability.

Further analyses focused on the influence of the Task Phase factor: Two contrasts were tested. The first contrast weighted pretask as 2 and the two posttask measures as -1 . It tested whether there was an overall difference between states measured prior to and following performance. The contrast was significant for task engagement, $F(1,62)=$ $15.7, p<.01, \eta_{\mathrm{p}}{ }^{2}=.2$, distress, $F(1,62)=11.52, p<.05$, $\eta_{\mathrm{p}}{ }^{2}=.16$, and worry, $F(1,62)=35.21, p<.01, \eta_{\mathrm{p}}{ }^{2}=.36$. Thus, performing the RoboFlag task tends to induce a multifaceted change in state. These state changes are summarized in Figure 4, which shows pretask levels of the DSSQ factors and the averaged posttask DSSQ scores from the HM and LM conditions. Table 2 shows that changes in state from pre- to posttask were in the same direction for each task condition.

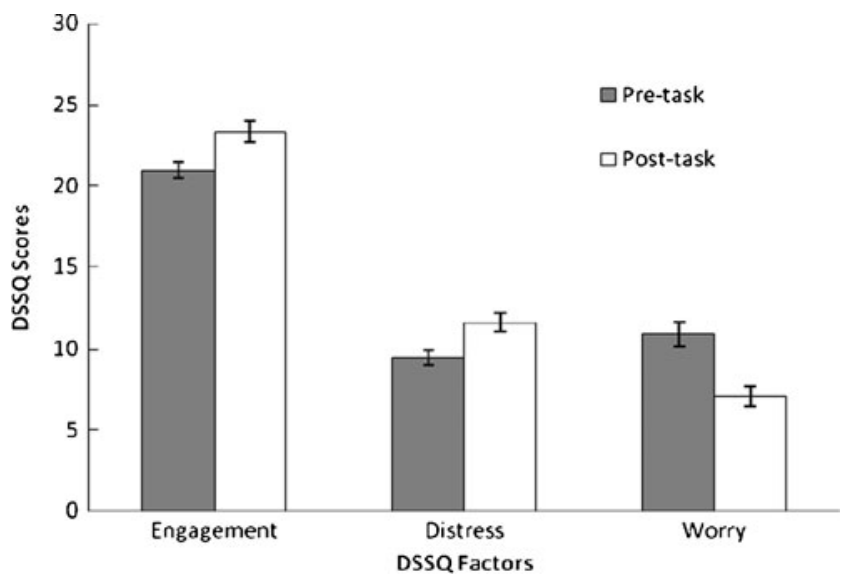

Fig. 4 Pre- and posttask DSSQ factor scores for engagement, distress, and worry. Error bars are standard errors
The second contrast weighted pretask as 0 , low uncertainty as 1 , and high uncertainty as -1 . It tested the effect of uncertainty within the two task conditions and was significant for distress, $F(1,62)=4.22, p<.05, \eta_{\mathrm{p}}^{2}=.06$, close to significance for engagement, $F(1,62)=3.24, p=.08$, $\eta_{\mathrm{p}}{ }^{2}=.05$, and nonsignificant for worry $(p>.05)$. In the LU condition, distress was higher, and there was also a trend toward reduced engagement, as is shown in Table 2.

\section{Perceived mental workload (NASA-TLX)}

Figure 5 depicts the workload profile of the RoboFlag task (i.e., mean workload ratings for the whole sample), both as a qualitative representation of the task demands and for comparative purposes with other tasks (Hart \& Staveland, 1988). Mental demands and effort were the most pronounced sources of workload. The effects of the task parameters on each rating were analyzed using 2 (uncertainty) $\times 2$ (maneuverability) mixed-model ANOVAs. Significant main effects of uncertainty were found in two of these analyses, for mental demands, $F(1,62)=4.34$, $p<.05, \eta_{\mathrm{p}}{ }^{2}=.06$, and temporal demands, $F(1,62)=4.71$, $p<.05, \eta_{\mathrm{p}}{ }^{2}=.07$. In the LU condition, the means for mental and temporal demands were $61.5(S D=24.9)$ and 49.8 $(S D=26.0)$, respectively. The corresponding means in the HU condition were $55.3(S D=29.5)$ and $42.3(S D=29.4)$. The means are averaged here across the two maneuverability groups, since there was no effect of this factor.

\section{Discussion}

The results of this study demonstrate the use of subjective measures to characterize stress and workload response to a complex performance task that captures some of the features of UAV operation, including substantial workload

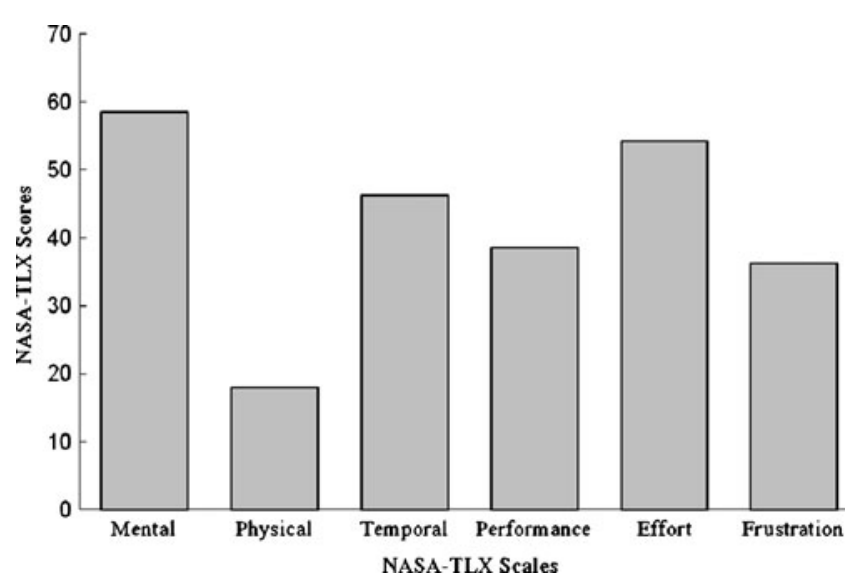

Fig. 5 Workload profile of the RoboFlag task: Mean scores averaged across all four conditions 
(Funke et al., 2006; Mouloua et al., 2003, Sterling \& Perala, 2007). The findings supported the utility of RoboFlag as the basis for a multivariate assessment methodology. Performing the task elicited a patterned subjective response, associated with elevations of both distress and task engagement, as well as reduced worry. The "input" (uncertainty) and "output" (maneuverability) manipulations provoked different patterns of response across multiple indicators (i.e., subjective stress, workload, and performance), reinforcing the suitability of this methodology for discriminating the impacts on the operator of differing sources of workload.

We obtained mixed support for specific hypotheses. The manipulations of uncertainty and maneuverability influenced performance as expected. The interactive effect of task parameters suggested that the combination of high uncertainty and low maneuverability made the robots particularly difficult for players to control. However, the effects of uncertainty and maneuverability on stress and workload indicators differed from initial expectation.

The results supported the multivariate approach to profiling task-induced stress (Matthews et al., 2002), in that performing the task appeared to enhance some aspects of subjective state (higher task engagement, lower worry), while also increasing distress, consistent with findings on other high-workload tasks (Matthews \& Campbell, 2010; Matthews et al., 2002; Matthews et al., 2006). A taskinduced reduction in worry is commonly found in experimental studies as attention is reallocated from taskirrelevant concerns to task processing (Matthews et al., 2002). The increase in task engagement contrasts with typical findings from studies of simple laboratory tasks (e.g., Matthews et al., 2006), in which engagement typically declines (e.g., vigilance) or remains constant (e.g., working memory). It may reflect the greater interest and challenge provided by working on a complex, gamelike task. Parsons, Warm, Nelson, Riley, and Matthews (2007) showed that increasing the operator's level of control over task events increases task engagement. Thus, synthetic task environments may have advantages over simple laboratory tasks as a methodology for investigating stress responses elicited by complex operational tasks.

The results did not support the predicted effects of the task parameters on workload and distress. Contrary to expectation, the low-uncertainty task produced higher levels of distress, mental demands, and temporal demands than did the high-uncertainty task. Players' ability to view more enemy robots in the low-uncertainty condition may have imposed greater mental demands. Although uncertainty is typically seen as a stress factor (e.g., Chen \& Hong, 2010), in the present context perhaps "ignorance is bliss." The effect of uncertainty observed in the present experiment contrasts with Parasuraman et al.'s (2005) finding that higher uncertainty increases workload. Changes to field of view may have nonlinear effects. Increasing the field of view in RoboFlag may decrease workload up to the point at which the field of view includes so many enemy robots that the player cannot process the information effectively, which is accompanied by an increased workload. The trend toward higher engagement in the low-uncertainty condition suggests that greater awareness of multiple enemy robots may also be somewhat challenging and motivating.

The findings may also have some more general applications beyond the immediate issue of UAV operation. When new technology leads to unexpected, adverse effects, one solution is to improve system design. For example, Stanton and Young (2005) suggested that automated systems in vehicles, such as adaptive cruise control, should display cues to the driver as to which other vehicles require attention. Multivariate assessment of a novel display, using a synthetic task environment, may serve to identify whether it elicits potentially dangerous stress, workload, or performance responses. The present findings on uncertainty effects demonstrated the relevance of this factor to display design. The wide field of view (low uncertainty) provides the operator with much taskrelevant information and improves performance. However, it also potentially overloads the operator and elevates distress. Thus, the display designer must negotiate the balance between providing sufficient information and avoiding overload. Multivariate assessment of alternate display designs may be useful in optimizing display characteristics.

Another issue in the operation of complex systems is the operator's ability to compensate for changes in task demands. For example, power plant operators develop a variety of strategies, including modifications to the control interface, in support of the multitasking necessary to maintain safe operations (Vicente, Mumaw, \& Roth, 2004). In some settings, adaptive coping carries a cost associated with increased stress; in others, cognitive strategy change does not entail such costs (see, e.g., Hockey, 2011). For example, in the present study, maneuverability impacted performance, but not stress and workload. Participants appeared to be capable of adapting their task strategies to variation in the control characteristics of the robots so that, although performance was impaired, they did not exert additional mental effort or experience elevated stress. The dissociation of stress and workload response from performance change shows that subjective measures of operator functional state add usefully to performance data in evaluating task demands. The assessment methodology permits evaluation of the strain imposed by strategy change; in other task environments, strategy change might carry significant stress/workload costs. 
Some limitations of the study must be acknowledged. RoboFlag provides a general test environment for investigating operator control of multiple simulated UAVs, but its demands differ considerably from those of real UAV operation. The utility of the methodology could also be tested against further workload parameters, such as the number of UAVs controlled by the operator (Hancock et al., 2007). Real operators, of course, have greater experience, training, and motivation than do undergraduate students. These factors may have a protective effect against stress, although previous longitudinal studies using the DSSQ have shown that task-induced stress responses persist over periods of weeks and months (Matthews \& Campbell, 2010; Matthews \& Falconer, 2000). The task duration was also fairly short, and changes in stress and workload might be seen over more extended operations. Further studies could extend the duration of the RoboFlag task in order to address the fatigue issues noted by Tvaryanas and MacPherson (2009). Further work might also investigate fine-grained strategy variations that may also influence workload and stress.

The RoboFlag methodology illustrates the general advantages of using synthetic task environments to represent the complex interplay of changing environmental characteristics and operator responses that are typical of a range of operational settings. An alternative methodology for stress assessment is to evaluate the responses of actual personnel operating a high-fidelity simulator or performing real missions. For some applications, realism may be essential, but the advantages of the RoboFlag methodology over real-life studies may be justified as follows: First, there are numerous potential sources of workload for the UAV operator (Mouloua et al., 2003; Sterling \& Perala, 2007). Testing the impact of each one of these in trained personnel is likely to be expensive and difficult to accomplish. The use of RoboFlag in undergraduate samples also readily affords the use of sample sizes of sufficient power to detect moderate effect. Second, RoboFlag studies may perform a useful filtering operation, in picking out poorly designed display characteristics or mission profiles at an early stage, without the need for a study of field personnel. Third, UAV operation is but one instance of various human factors settings in which robots are controlled remotely. RoboFlag studies may provide a methodology for building a general account of influences on stress and workload in robot operation, which may then be tailored toward more specific applications. Thus, we advocate a general methodology of using RoboFlag (and similar synthetic task environments) to identify potential stress and workload issues for UAV operations, which may be followed by carefully targeted, smaller-sample studies with trained personnel to confirm generalization of the laboratory findings to operational practice. The multivariate assessment methodology may also be more widely applicable to a variety of complex operational tasks in industrial, transportation, and military/ security contexts.

\section{References}

Boyes, M. E., \& French, D. J. (2010). Neuroticism, stress, and coping in the context of an anagram-solving task. Personality and Individual Differences, 49, 380-385.

Campbell, M., Rafffaello, D., Schneider, D., Chaudhry, A., Waydo, S., Sullivan, J., et al. (2005). RoboFlag games using system based hierarchical control. Cornell RoboFlag. Retrieved February 20, 2010, from www.cds.caltech.edu/ waydo/papers/ACC03.pdf

Ceaparu, I., Lazar, J., Bessiere, K., Robinson, J., \& Shneiderman, B. (2004). Determining causes and severity of end-user frustration. International Journal of Human-Computer Interaction, 17, 333 356.

Chen, C. Y., \& Hong, Y. (2010). Intolerance of uncertainty moderates the relation between negative life events and anxiety. Personality and Individual Differences, 49, 49-53.

Cooke, N. J., \& Shope, S. M. (2004). Designing a synthetic task environment. In S. G. Schiflett \& L. R. Elliott (Eds.), Scaled worlds: Development, validation, and applications (1st ed., pp. 263-278). Aldershot: Ashgate.

Cummings, M. L., \& Guerlain, S. (2007). Developing operator capacity estimates for supervisory control of autonomous vehicles. Human Factors, 49, 1-15.

D'Andrea, R., \& Babish, M. (2003). The RoboFlag testbed. In Proceedings of the American Control Conference, 22nd Annual Meeting (pp. 656-660). Dayton, OH: American Automatic Control Council.

Funke, G. J., Galster, S. M., Nelson, W. T., \& Dukes, A. W. (2006, June). Instant messaging and team performance in a simulated command and control environment. Paper presented at the 2006 CCRTS, San Diego, CA. Retrieved October 10, 2010, from www.dodccrp.org/events/2006_CCRTS/html/papers/092.pdf

Funke, G. J., Matthews, G., Warm, J. S., \& Emo, A. (2007). Vehicle automation: A remedy for driver stress? Ergonomics, 50, 1302-1323.

Hancock, P. A., \& Szalma, J. L. (2003). Operator stress and display design. Ergonomics in Design, 11, 13-18.

Hancock, P. A., Mouloua, M., Gilson, R. D., Szalma, J., \& Oron-Gilad, T. (2007). Is the UAV control ratio the right question? Ergonomics in Design, 15, 30-31.

Hart, S. G., \& Staveland, L. E. (1988). Development of a multidimensional workload scale: Results of empirical and theoretical research. In P. A. Hancock \& N. Meshkati (Eds.), Human mental workload (pp. 139-183). Amsterdam: North-Holland.

Hockey, G. R. J. (2011). A motivational control theory of cognitive fatigue. In P. L. Ackerman (Ed.), Cognitive fatigue: Multidisciplinary perspectives on current research and future applications (pp. 167187). Washington, DC: American Psychological Association.

Martin, E., Lyon, D. R., \& Schreiber, B. T. (1998). Designing synthetic tasks for human factors research: An application to uninhabited air vehicles. In Proceedings of the Human Factors and Ergonomics Society, 42nd Annual Meeting (pp. 123-127). Santa Monica, CA: Human Factors and Ergonomics Society.

Matthews, G., \& Campbell, S. E. (2010). Dynamic relationships between stress states and working memory. Cognition and Emotion, 24, 357-373.

Matthews, G., \& Falconer, S. (2000). Individual differences in taskinduced stress in customer service personnel. In Proceedings of the Human Factors and Ergonomics Society, 44th Annual Meeting (pp. 145-148). Santa Monica, CA: Human Factors and Ergonomics Society. 
Matthews, G., Campbell, S. E., Falconer, S., Joyner, L. A., Huggins, J., Gilliland, K., et al. (2002). Fundamental dimensions of subjective state in performance settings: Task engagement, distress, and worry. Emotion, 2, 315-340.

Matthews, G., Emo, A. K., \& Funke, G. J. (2005). A short version of the Dundee Stress State Questionnaire. Paper presented at the Twelfth Meeting of the International Society for the Study of Individual Differences, Adelaide, Australia.

Matthews, G., Emo, A. K., Funke, G., Zeidner, M., Roberts, R. D., Costa, P. T., Jr., et al. (2006). Emotional intelligence, personality, and task-induced stress. Journal of Experimental Psychology. Applied, 12, 96-107.

Matthews, G., Warm, J. S., Reinerman, L. E., Langheim, L. K., \& Saxby, D. J. (2010). Task engagement, attention and executive control. In A. Gruszka, G. Matthews, \& B. Szymura (Eds.), Handbook of individual differences in cognition: Attention, memory and executive control (pp. 205-230). New York: Springer.

McCarley, J. S., \& Wickens, C. D. (2005). Human factors implications of UAVs in the national airspace (Tech. Rep. AHFD-05-05/ FAA-05-1). Savoy, IL: University of Illinois, Aviation Human Factors Division.

Mouloua, M., Gilson, R., \& Hancock, P. (2003). Human-centered design of unmanned aerial vehicles. Ergonomics in Design, 11, 6-11.

Mouloua, M., Gilson, R., Krig, J., \& Hancock, P. (2001). Workload, situational awareness and teaming issues for UAV/UCAV operations. In Proceedings of the Human Factors and Ergonomics Society, 45th Annual Meeting (pp. 162-165). Santa Monica, CA: Human Factors and Ergonomics Society.

Parasuraman, R., Galster, S., \& Miller, C. (2003). Human control of multiple robots in the RoboFlag simulation environment. In Proceedings of the IEEE International Conference on Systems, Man and Cybernetics (Vol. 4, pp. 3232-3237). Piscataway, NJ: IEEE Press.

Parasuraman, R., Galster, S., Squire, P., Furukawa, H., \& Miller, C. (2005). A flexible delegation-type interface enhances system performance in human supervision of multiple robots: Empirical studies with RoboFlag. IEEE Transactions on Systems, Man, and Cybernetics. Part A: Systems and Humans, 35, 481-493.

Parsons, K. S., Warm, J. S., Nelson, W. T., Riley, M., \& Matthews, G. (2007). Detection-action linkage in vigilance: Effects on workload and stress. In Proceedings of the Human Factors and Ergonomics Society, 51st Annual Meeting (pp. 1291-1295). Santa Monica, CA: Human Factors and Ergonomics Society.

Saxby, D. J., Matthews, G., Hitchcock, E. M., \& Warm, J. S. (2007). Development of active and passive fatigue manipulations using a driving simulator. In Proceedings of the Human Factors and Ergonomics Society, 51st Annual Meeting (pp. 1237-1241). Santa Monica, CA: Human Factors and Ergonomics Society.

Schipani, S. P. (2003). An evaluation of operator workload during partially-autonomous vehicle operations. Paper presented at Performance Metrics for Intelligent Systems (PerMIS) 2003, Gaithersburg, MD.

Squire, P., Trafton, G., \& Parasuraman, R. (2006). Human control of multiple unmanned vehicles: Effects of interface type on execution and task switching times. Retrieved December 24, 2010, from www.nrl.navy.mil/aic/iss/pubs/squire.hri06.pdf

Stanton, N., \& Young, M. S. (2005). Driver behavior with adaptive cruise control. Ergonomics, 48, 1294-1313.

Sterling, B. S., \& Perala, C. H. (2007). Workload, stress, and situation awareness of soldiers who are controlling unmanned vehicles in future urban operations (Tech. Rep. No. ARL-TR-4071). Washington, DC: Army Research Laboratory.

Tvaryanas, A. P., \& MacPherson, G. D. (2009). Fatigue in pilots of remotely piloted aircraft before and after shift work adjustment. Aviation Space and Environmental Medicine, 80, 454-461.

van Erp, J. B. F. (2000). Controlling unmanned vehicles: The human factors solution. In Advances in vehicle systems concepts and integration: RTO Meeting Proceedings 44 (Tech. Rep. No. RTOMP-44, pp. B8.1-B8.12). Neuilly-sur-Seine Cedex, France: NATO Research and Technology Organization.

van Erp, J. B. F., \& van Breda, L. (1999). Human factors issues and advanced interface design in maritime unmanned aerial vehicles: A project overview (TNO Report TM-99-A004). Amsterdam: TNO.

Veverka, J., \& Campbell, M. (2003). Experimental study of information load on operators in semi-autonomous systems. Cornell RoboFlag. Retrieved February 20, 2010, from http:// roboflag.mae.cornell.edu/publications/VeverkaAIAA.pdf

Vicente, K. J., Mumaw, R. J., \& Roth, E. M. (2004). Operator monitoring in a complex dynamic work environment: A qualitative cognitive model based on field observations. Theoretical Issues in Ergonomics Science, 5, 359-384.

Wickens, C., \& Hollands, J. (2000). Engineering psychology and human performance. Upper Saddle River: Prentice Hall.

Worsch, P., Borky, J., Gabriel, R., Heiser, W., Swalm, T., \& Wong, T. (1996). UAV technologies and combat operations: Vol. 2 (Tech. Rep. No. SAB-TR-96-01). Washington, DC: U.S. Air Force Scientific Advisory Board.

Young, M. S., \& Stanton, N. A. (2007). What's skill got to do with it? Vehicle automation and driver mental workload. Ergonomics, 50, 1324-1339. 